\title{
Effects of Scleral Contact Lenses for Keratoconus Management on Visual Quality and Intraocular Pressure
}

\section{Martina Formisano (D) \\ Federica Franzone \\ Ludovico Alisi \\ Santino Pistella \\ Leopoldo Spadea (iD}

Eye Clinic, Department of Sense Organs, University of Rome Sapienza, Policlinico

Umberto I, Rome, Italy
Correspondence: Leopoldo Spadea Eye Clinic, Policlinico Umberto I, "Sapienza" University of Rome, Via Benozzo Gozzoli 34 Rome 00142, Italy

Tel +039065193220

Fax +3906 8865 7818

Email leopoldo.spadea@uniromal.it
This article was published in the following Dove Press journal: Therapeutics and Clinical Risk Management

Purpose: To evaluate the visual acuity level achieved by scleral contact lens (CL) fitting in patients affected by keratoconus and to evaluate possible intraocular pressure (IOP) changes during the scleral CL wear using a transpalpebral tonometer.

Methods: In a prospective case series a comparison was made between visual acuity obtained with glasses, RGP and $16.8 \mathrm{~mm}$ diameter scleral CL in 30 consecutive patients affected by keratoconus. IOP was tested during scleral CL wear by transpalpebral Diaton Tonometer (DT) and also by Goldmann Applanation Tonometer (GAT) before and after scleral CL wear.

Results: The mean $\log$ MAR visual acuity improved from $0.2 \pm 0.25 \mathrm{SD}$ with glasses and 0.1 $\pm 0.02 \mathrm{SD}$ with RGP, to $-0.002 \pm 0.041 \mathrm{SD}$ when using the scleral CL $(p<0.05)$. The mean IOP value before scleral $\mathrm{CL}$ wear was $12.93 \mathrm{mmHg} \pm 2.20 \mathrm{SD}$ when measured with GAT and $7.85 \mathrm{mmHg} \pm 2.27 \mathrm{SD}$ when measured with DT. During scleral CL wear, IOP was assessed through DT, with a mean value of $8.86 \mathrm{mmHg} \pm 2.36 \mathrm{SD}$; values were stable after 8 hours of scleral CL use. Immediately after scleral CL removal, the mean IOP measured with GAT was $12.85 \mathrm{mmHg} \pm 2.40 \mathrm{SD}$ and the mean IOP measured with DT was $7.66 \mathrm{mmHg} \pm 1.88 \mathrm{SD}$. Therefore, during scleral CL wear, it was evidenced a small but statistically significant increase of the mean IOP value $(1.01 \mathrm{mmHg} ; p<0.01)$, with a reversion to values prior to application when scleral CL was removed.

Conclusion: Scleral CLs remarkably improved visual acuity in keratoconus patients when compared to glasses or RGP contact lenses. Even if it was evidenced a small increase of the mean IOP value during their wear, it may not be significant in otherwise healthy eyes. Statistical analysis demonstrated good agreement between GAT and DT but their numerical values presented a constant gap, that should be taken into account when there is a need to relate the DT values to the reference ones.

Keywords: keratoconus, higher-order aberrations, IOP, scleral contact lens

\section{Introduction}

Keratoconus is a non-inflammatory corneal disease that causes a gradual and progressive corneal ectasia. The disease typically involves both eyes. ${ }^{1}$ In keratoconus, all cornea layers, particularly the stroma, are characterized by a disruption in the arrangement of collagen fibrils, alterations in proteoglycans expression, and unstable interconnectivity of collagen lamellae, with varying degrees of severity. ${ }^{2}$ Changes in stromal biomechanics result in paracentral steepening of the cornea, apical thinning, and conic shaping of the cornea. ${ }^{3}$ The displaced optic zone leads to 
irregular astigmatism, significant coma aberration, and a remarkable reduction in the quality of vision. ${ }^{4}$

Traditional sphero-cylindrical spectacles do not provide significant visual improvement in keratoconus patients, due to higher-order aberrations (HOAs) typical of the keratoconic cornea. Consequently, when a high number of HOAs appear in keratoconus patients, rigid gas permeable (RGP; corneal, corneoscleral, and scleral) contact lenses are suggested as soon as they create a more regular refractive surface. ${ }^{4}$ However, corneal RGP lenses may not be suitable for advanced corneal irregularity and can be associated with corneal scarring at the point of contact between the lens and cornea. ${ }^{5}$

Piggyback contact lenses, intended as rigid gaspermeable lenses that are fitted over soft contact lenses, are suggested for people who cannot tolerate rigid gas permeable lenses, but they are more expensive and have complex maintenance and storage. ${ }^{6}$

Scleral lenses are large-diameter rigid gas-permeable lenses, which vault over the cornea and limbal area and rest on the conjunctival tissue overlying the sclera, which provides a major comfort during their use. The midperipheral and peripheral parts of the lenses are customizable to improve stabilization. Even if scleral CLs share the same composition as RGP contact lenses, the entrapment of a thick layer of fluid beneath the scleral contact lens alters the relationship between the cornea and the lens, and also provide relief in case of dry eye. ${ }^{7}$

Scleral CLs can have different diameters but generally, inferior diameter scleral CLs (previously referred to as mini-scleral CL) have easier handling ${ }^{8}$ and are more proximal to structures involved in the aqueous humour outflow than larger diameter lenses. ${ }^{6}$

The indication for surgery is usually due to contact lenses intolerance or a limitation of the best spectacles or contact lenses-corrected visual acuity. ${ }^{9}$ Nonetheless, high levels of aberrations may not be eliminated with surgery, and have been reported in keratoconus patients treated with intrastromal corneal ring segments, cross-linking, radial keratotomy and lamellar or penetrating keratoplasty. ${ }^{10,11}$ Contact lenses also may be needed after surgical intervention for keratoconus to address the HOAs still present.

In this study, we evaluated the effects of a $16.8 \mathrm{~mm}$ diameter scleral CL wear in keratoconus eyes in terms of visual acuity and intraocular pressure, the latter measured through a transpalpebral tonometer.

\section{Methods}

The present study was designed as a prospective case series. The study was approved by the ethical board of the Sapienza University of Rome, Umberto I Hospital, and was conducted in accordance with the tenets of the Declaration of Helsinki. Written informed consent was obtained by all the patients. The patients attended a dedicated keratoconus department in our university hospital (Policlinico Umberto I, Rome) for 1-year (from June 1st, 2018 to June 1st, 2019). The study included 60 eyes of 30 consecutive patients ( 9 females and 21 males) with a mean age of $38.4 \pm 11.8$ years (range $26-62 y$ ). All the patients were affected by different grades of keratoconus in both eyes and they were visually unsatisfied after correction with traditional spectacles or corneal RGP (Table 1). We classified keratoconus using Krumeich classification: keratometric measurements $<48 \mathrm{D}$ were graded as "stage 1" keratoconus, measurements $<53 \mathrm{D}$ were graded as "stage 2" keratoconus, measurements $>53$ D were classed as "stage 3" keratoconus, and measurements $>55 \mathrm{D}$ were graded as the ultimate stage, "stage 4 ". ${ }^{12}$ Subjects were free of any systemic or concomitant ocular disease. They were not known as being at risk for glaucoma and had no familial history for this pathology, and they had no previous eye surgeries. Subjects who were contact lens wearers were instructed to stop wearing contact lens at least ten days before the evaluation day. Objective and subjective refraction was conducted with trial frame lenses under standardized room and chart illumination conditions by one of the authors (MF). The distance corrected visual acuity (DCVA) was measured using an ETDRS chart and given as a logarithm of minutes of arc resolution (logMAR). Baseline findings for anterior central and posterior corneal curvature, corneal pachymetry, iridocorneal angle, as well as anterior chamber depth and volume, using different maps were taken with Precisio

Table I Eyes Divided in 4 Groups Based on Krumeich Classification

\begin{tabular}{|l|l|l|l|l|}
\hline $\mathbf{n = 6 0}$ & $\begin{array}{l}\text { K } \\
\text { 48D } \\
\text { Stage } \\
\text { I }\end{array}$ & $\begin{array}{l}\text { K >48 } \\
\text { <53D } \\
\text { Stage } \\
\text { II }\end{array}$ & $\begin{array}{l}\text { K >53D } \\
\text { <5D } \\
\text { Stage III }\end{array}$ & $\begin{array}{l}\text { K >55D } \\
\text { Stage } \\
\text { IV }\end{array}$ \\
\hline $\begin{array}{l}\text { Number of eyes with } \\
\text { keratoconus at } \\
\text { a specific stage }\end{array}$ & 5 & 14 & 18 & 23 \\
\hline
\end{tabular}

Abbreviations: $\mathrm{n}$, sample size (eyes studied); K, keratometric measurements; D, diopters. 
2 tomographer (iVIS Technologies, Taranto, Italy). IOP values before scleral CL wear were assessed by both GAT (Haag-Streit AT900, USA) and DT (BICOM Inc, Long Beach, NY, USA), to have comparable measurements before and after the contact lenses wear. ${ }^{13}$

Then, a customized scleral contact lens (Medlac $16.8 \mathrm{~mm}$ diameter; MedLac, Avellino, Italy) was prescribed to the 30 enrolled subjects. These Medlac lenses were chosen because of their ease of use given by their intermediate diameter, which is essential for patients requiring daily usage. Moreover, these lenses allowed us to maintain a safety distance of $2 \mathrm{~mm}$ between the corneal limbus and the lens landing zone, to avoid a possible compression of the angle structures.

Patients with a corneal diameter greater than $12.30 \mathrm{~mm}$ or less than $11.80 \mathrm{~mm}$ have been excluded since the manufacturer indicates the $16.80 \mathrm{~mm}$ diameter suitable for medium-sized horizontal visible iris diameter (HIVD). The $16.8 \mathrm{~mm}$ overall diameter scleral CL not fenestrated were used for this study made by Tisilfocon A, with DK of 180 , and a flexure modulus of $1341 \mathrm{Mpa}$. Lenses were manufactured with $220 \mu \mathrm{m}$ center thickness. The landing zone angle was assessed through AS-OCT. It ranged from $48^{\circ}$ to $52^{\circ}$. The landing zone angle was established to avoid edge compression or impeachment in the conjunctiva tissue and to avoid excessive edge lift to the sclera portion. An experienced examiner (BS) performed all fitting following the manufacturer's guide. Subjects with a difference between HIVD and vertical visible iris diameter (VVID) of more than $0.5 \mathrm{~mm}$, were fitted with scleral LC Conica Evolution design, with an ellipticalshaped corneal chamber; allowing to control the distance between the lens landing and the limbus in each sector. Scleral CLs were inserted with preservative-free saline and sodium fluorescein and then assessed using a slit-lamp biomicroscope. The relationship between the central lens and the cornea was evaluated, checking that the lens was without air bubbles. Lenses with smaller or larger back optic zone radii of curvature were tested progressively until the highest point of the cornea (apex) showed a light feather apical clearance (Figure 1). Once centering was achieved, the settling of the lens on the conjunctiva was observed to avoid over-pressure on the conjunctival vessels, a settling of the scleral portion on the conjunctiva was considered normal within a range of $100-150 \mu \mathrm{m}$, evaluated by the anterior segment module of Spectralis OCT (Heidelberg engineering Inc, USA). This procedure was followed by a final slit-lamp examination to assess

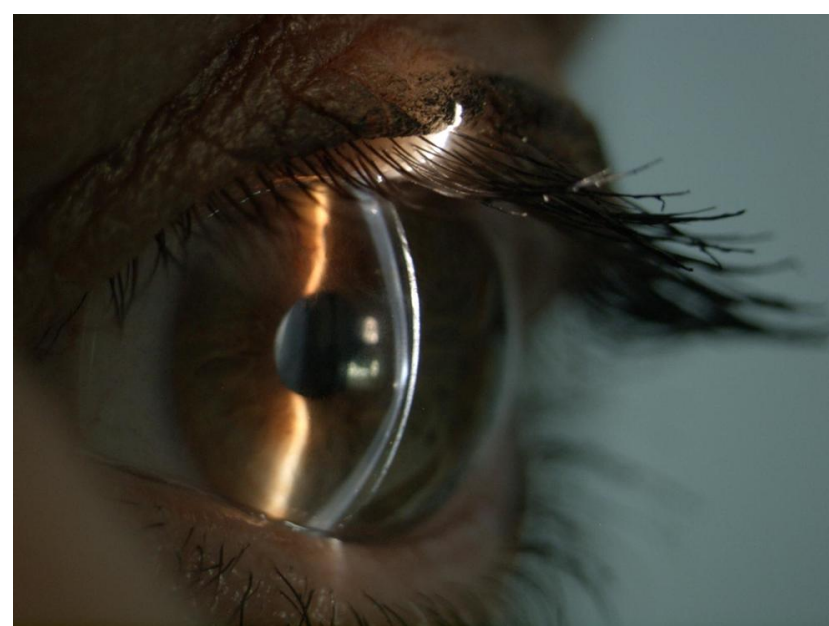

Figure I Slit-lamp examination of a patient's eye fitting a scleral lens (Medlac $16.8 \mathrm{~mm}$ diameter). It's visible a thin tear layer between the posterior lens surface and the corneal apex.

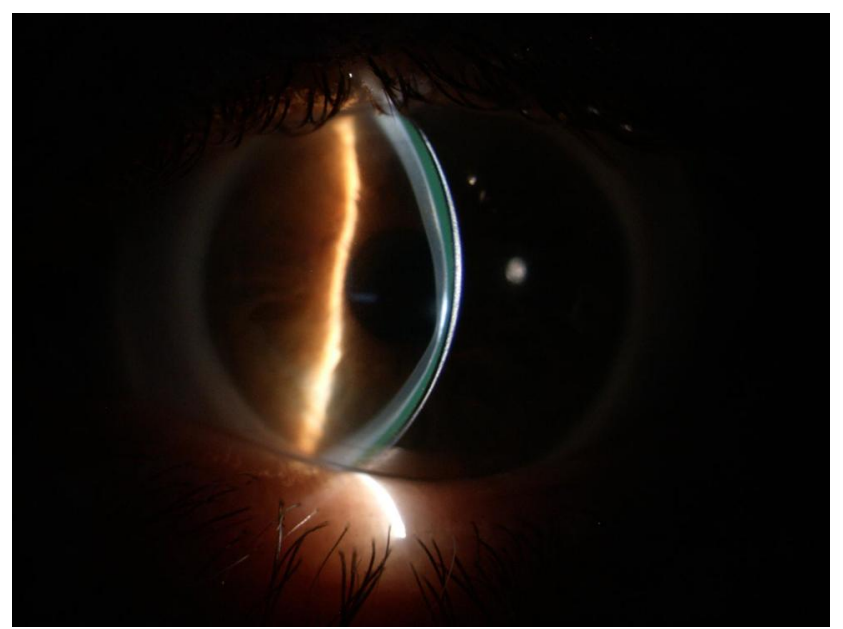

Figure 2 Scleral lenses were inserted with preservative-free saline and sodium fluorescein, which helped the analysis of the tear allocation under the lens. The colored tear layer is homogeneous and air-bubbles free.

ocular health (Figure 2). Lissamine Green stain was used to check any signs of conjunctival distress during lens wear as well as after the lens removal.

The intraocular pressure (IOP) during scleral CL wear was evaluated through the DT at baseline and 8 hours after. Comfort and visual acuity with the scleral CL were documented. The scleral lenses were then carefully removed by using a small plunger to avoid inducing excessive pressure on the ocular surface during the process. ${ }^{14}$ Then, just after lens removal, we measured again the IOP with both GAT and DT.

The Diaton tonometer has a U-shaped tip designed to adapt to the eyelid and obtain IOP measurements through a freely moving rod inside the tonometer, which falls 
freely onto the elastic surface of the eyeball through the eyelid. Thus, the instrument measures the resistance of the eyelid, the conjunctiva, and sclera through the backward acceleration after interaction with the sclera via the eyelid and converts it to an estimate of intraocular pressure. The Diaton automatically displays the average measurement of up to 6 readings. The measurements in our study were taken with the examiner and the patient facing the same direction. During the DT, the patients were sitting with their heads reclined, looking at an index finger to obtain primary gaze fixation. The upper eyelid was retracted so that the lid margin was $1 \mathrm{~mm}$ above the superior limbus and the tonometer's tip was placed perpendicularly onto the tarsal plate, at about $2 \mathrm{~mm}$ behind the superior lid margin, careful to avoid placing the tonometer above the scleral lens when applied. When the tip of the tonometer touched the eyelid correctly, the measurement was noted automatically. The tonometer is tilted back from vertical to reset the scale for each of the two following measurements after which the average of the three was displayed digitally on the screen. In this study, all DT measures were made by the same experienced observer to reduce the variability in the measurement process. ${ }^{15,16}$

Statistical analysis with Student's $t$-test for paired data was performed to investigate the effect on IOP of scleral $\mathrm{CL}$ repeating the measurement before, during and after the application. Bland-Altman method was repeatedly employed to evaluate the level of agreement between DT and GAT before scleral CL wearing, and after their removal (95\% limits of agreement was calculated as mean difference $\pm 1.96 \mathrm{SD}$ ). All analyses were performed averaging measurement obtained in both eyes.

\section{Results}

The mean $\operatorname{logMAR}$ visual acuity improved from 0.2 $\pm 0.25 \mathrm{SD}$ with glasses and $0.1 \pm 0.02 \mathrm{SD}$ with RGP to $-0.002 \pm 0.041 \mathrm{SD}$ in all the eyes wearing scleral CL $(p<0.05)$. All the patients found the scleral $\mathrm{CL}$ to be comfortable with a mean daily wear time of more than 6 hours. Average IOP values before, during, and after scleral $\mathrm{CL}$ wear are listed in Table 2. The high $p$-value shows that there is not a significant difference in IOP before lens wearing and after their removal for both GAT $(p=0.45)$ and DT $(p=0.31)$.

$t$-test performed on DT results before and during the lens wearing showed a significant increase in IOP $(p$-value $<0.01)$. Similar results were obtained when comparing IOP measured with DT during and after lens removal $(p$-value $<0.01)$. These
Table 2 Average IOP Obtained with GAT and DT Before, During and After Scleral CL Wear. Results are Expressed in Millimeters of Mercury Along with the Standard Deviation Value

\begin{tabular}{|l|l|l|}
\hline $\begin{array}{l}\mathbf{n}=\mathbf{6 0} \text { Eyes Affected } \\
\text { by Keratoconus }\end{array}$ & $\begin{array}{l}\text { GAT } \\
\text { (Mean } \\
\text { IStandard } \\
\text { Deviation) }\end{array}$ & $\begin{array}{l}\text { DT } \\
\text { (Mean } \\
\text { IStandard } \\
\text { Deviation) }\end{array}$ \\
\hline Without scleral CL & $12.93 \pm 2.20$ & $7.85 \pm 2.27$ \\
$\begin{array}{l}\text { During scleral CL wear } \\
\text { After scleral CL removal }\end{array}$ & $\begin{array}{l}\text { Not evaluable } \\
12.85 \pm 2.40\end{array}$ & $\begin{array}{l}8.86 \pm 2.36 \\
7.66 \pm 1.88\end{array}$ \\
\hline
\end{tabular}

Abbreviations: $n$, sample size; GAT, Goldmann applanation tonometry; DT, Diaton tonometry; $\mathrm{CL}$, contact lenses.

results indicate that scleral CLs induce a small but statistically significant DT increase in IOP. Using the Bland-Altman method, the upper and lower limits of agreement between DT and GAT were found to be +7.8 and +2.5 , respectively, before contact lens wearing. Upper and lower limits of agreement between DT and GAT were found to be +8.4 and +1.8 , after lens removal (Figure 3).

\section{Discussion}

The efficiency of scleral lenses in correcting aberrations due to keratoconus is well demonstrated by many studies. $^{6,8,17-19}$ In this study, our patients achieved a significantly better visual acuity with scleral CL when compared with spectacles or RGP contact lenses, providing an additional non-surgical option for the treatment of the pathology.

Besides, comfort was one of the primary advantages of scleral CL. Indeed, all the patients experienced relief from dryness and were able of tolerating at least a scleral $\mathrm{CL}$ wear of 6 hours daily. Scleral CLs regularized the corneal surface by vaulting the irregular keratoconic cornea with their surface and the tear reservoir underlying the lens. Differently, RGP contact lenses do not create a tear reservoir which vaults the cornea and their contact with the cornea, could compromise visual acuity and contact lenses tolerance. $^{5}$

IOP changes in these patients wearing scleral CL were also investigated. Unlike soft lenses, scleral CL compress and settle into conjunctival tissue. During each blink, the pressure from the lid may press the lens farther into the conjunctiva. ${ }^{20}$ In general, large-diameter scleral lenses $(\geq 18.0 \mathrm{~mm}$ in diameter) have relatively wide haptics or landing zones (up to $2 \mathrm{~mm}$ wide), with a broader distribution of the lens-bearing force on the eye. Thus, large-diameter scleral lenses may be less likely to compress any structures within or adjacent to the anterior 

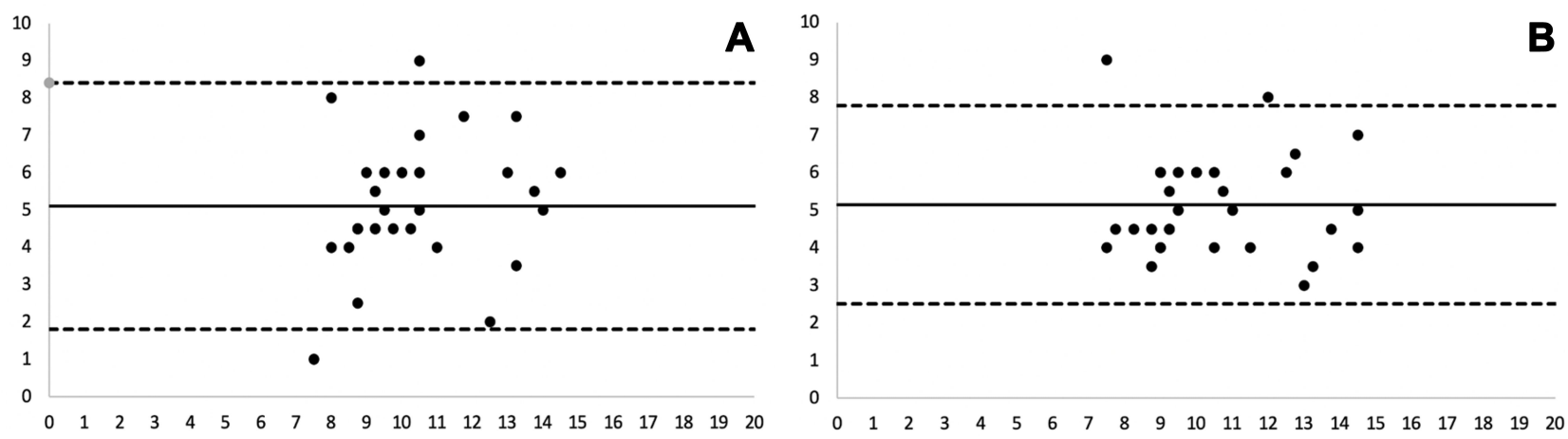

Figure 3 Bland-Altman plot on Diaton tonometry vs Goldmann tonometry, before the scleral lens application (A) and after their removal (B). Dotted black lines show upper limits of agreement (ULOA) and lower limits of agreement (LLOA). Continuous black line shows mean difference. (A) mean: +5.I5, ULOA: +7.8, LLOA: +2.5; (B) mean: +5. I0, ULOA: +8.4 LLOA: + 1.8. Diff: difference. Results are shown in $\mathrm{mmHg}$.

chamber angle. However, small-diameter scleral lenses (14.0 to $16.5 \mathrm{~mm}$ in diameter) generally have limited haptic widths and diameters, and they contact the conjunctiva closer to the limbus than do the larger lenses. The concentration of the bearing surface over a smaller area close to the limbus may presumably compress structures responsible for aqueous humor outflow as the Schlemm canal, the collector channels, or the episcleral veins and consequently increase intraocular pressure. ${ }^{20}$ Nonetheless, it is hypothesized that the functional diameter and the chord where the lens lands on the conjunctiva, are more important than the overall lens diameter. ${ }^{2}$ Compression of the structures of the anterior chamber angle could be indirectly related to signs of tight-fitting in the periphery such as blanching of the conjunctival blood vessels, conjunctival flush and marked suction between the lens and the conjunctival tissue that is difficult to break on lens removal. ${ }^{22}$ Ideally, well-fitted scleral lenses, which do not create excessively tight bearing zones as they settle, should not significantly elevate IOP. ${ }^{23}$ Apart from the degree of lens fit tightness, any IOP elevation associated with scleral fittings appears to be linked to individual patient characteristics. For example, eyes with reduced scleral thickness and/or rigidity appear likely to be more easily applanated at zones of tight scleral lens bearing. ${ }^{16}$ Anyway, eyes that are susceptible to baropathic diseases such as glaucoma, axial myopia, and corneal thinning diseases, such as keratoconus, could have pathological adverse events due to scleral CLs induced elevation of IOP. ${ }^{22}$ In literature, there are many studies regarding the relationship between scleral lens wear and IOP but results and methods range widely. Indeed, some studies conducted on lenses with a diameter between $15 \mathrm{~mm}$ and $18 \mathrm{~mm}$, did not find a significant rise in IOP during the SLs wear using a pneumotonometer ${ }^{20}$ or after the scleral CLs wear, using GAT, a Tonopen, or a non-contact tonometer. ${ }^{24-26}$ Differently, other authors found a rise in IOP (average of $5 \mathrm{mmHg}$ ) following scleral lens removal (diameter between $14.6 \mathrm{~mm}$ and $18 \mathrm{~mm}$ ) using GAT, DT, or Icare tonometer and during scleral CLs wear using DT or a rebound tonometer. ${ }^{21,27-29}$

Authors who found an IOP elevation consequently to scleral CL application supposed that such findings might be a consequence of increased susceptibility to scleral lensinduced IOP elevation due to having thinner scleral tissue. Indeed, a thinner sclera would be easier to applanate or indent by bearing areas of scleral lenses. Tight fittings might also explain such effects. Any rise in IOP may also depend on aqueous drainage facility, which might be restricted in some individuals. In addition, aqueous drainage may be compromised by lenses with bearing areas at the limbus. ${ }^{20}$

In the present study, we found a small $(1.01 \mathrm{mmHg})$ but statistically significant increase of the IOP measured with DT during the scleral CL wearing. We decided to use DT because, even if GAT represents the gold standard in the IOP measurement, it does allow IOP measurement while wearing scleral contact lenses, and measuring IOP after lens removal can only be indirectly relevant to lens-in situ IOP. ${ }^{30}$ The Diaton tonometer is a handheld device capable of measuring IOP through the upper eyelid, avoiding corneal contact. ${ }^{31}$ However, the transpalpebral tonometer is a novel and still not well-standardized technique to measure IOP in eyes wearing scleral CL. For the Diaton, the manufacturer describes a possible error of $\pm 2 \mathrm{mmHg}$ for eyes with IOP between 5 and $20 \mathrm{mmHg}$, and an error of $10 \%$ of the current IOP for IOP between 20 and $60 \mathrm{mmHg} .^{21,26}$ Even if there was good agreement between DT and GAT, we found that DT usually gives a lower IOP value (about $5 \mathrm{mmHg}$ ) compared to GAT results. From a clinical perspective, the differences are significant; indeed, it is not possible to relate values obtained with DT to reference values. Other studies 
reported poor agreement between DT and $\mathrm{GAT}^{32}$ under or overestimation of the $\mathrm{IOP}^{33}$ and a significant influence of the positioning on the IOP measurement with the DT. ${ }^{34}$ A moderate correlation between AT and DT was found when used on a normal eye in a study ${ }^{35}$ and when corrected for central corneal thickness in another study. ${ }^{31}$

\section{Conclusions}

Scleral CLs could be successfully used to treat corneal diseases such as keratoconus. They give a valid alternative to surgery with quality of vision and comfort superior to other actual devices. Even if $16.8 \mathrm{~mm}$ diameter scleral CLs have easier handling than larger diameter scleral CLs, their landing zone is closer to structures involved in the aqueous humor outflow. We demonstrated that well-fitted scleral CLs, checked by AS-OCT and biomicroscopic examination, do not usually cause any signs of distress on the eye, rendering unlikely a compression of the structures of the anterior chamber angle.

The IOP values acquisition during scleral CL wear has been possible thanks to a new transpalpebral tonometer. Even if the methodic is new and still not standardized, its parallel use to GAT before and after scleral CL use allowed a better interpretation of the results. Statistical analysis showed good agreement between the two instruments but a constant numeric difference between values obtained with GAT and DT. This should be considered when relating DT values to reference ones.

The DT showed a small but significant $(1.01 \mathrm{mmHg}$; $\mathrm{p}<0.01$ ) raise of the IOP during scleral CL wear. We believe that this small raise is unlikely to cause any glaucomatous damage in the eyes otherwise normal. Moreover, changes in the DT position should be occurred trying to avoid contact with the part of the superior lid overlying the scleral CL. Finally, it is recommendable for a proper selection and follow-up of the patients during the use of the scleral CLs. More studies with a wider casuistry are also needed.

\section{Acknowledgment}

Presented in part at the "International Summit of Specialty Contact Lenses (ISSCL)”, Rome (Italy) 8-10 November 2019.

\section{Funding}

This research did not receive any specific grant from funding agencies in the public, commercial, or not-for profit sectors.

\section{Disclosure}

The authors report no conflicts of interest in this work.

\section{References}

1. Chang HY, Chodosh J. The genetics of keratoconus. Semin Ophthalmol. 2013;28(5-6):275-280. doi:10.3109/08820538.2013 .825295

2. Alkanaan A, Barsotti R, Kirat O, Khna A, Almubrad T, Akhtar S. Collagen fibrils and proteoglycans of peripheral and central stroma of the keratoconus cornea - Ultrastructure and 3D transmission electron tomography. Sci Rep. 2019;9:1-11. doi:10.1038/s41598-019-56529-1

3. Jinabhai AN. Customised aberration-controlling corrections for keratoconic patients using contact lenses. Clin Exp Optom. 2020;103 (1):31-43. doi:10.1111/cxo.12937

4. Montalt JC, Porcar E, España-Gregori E, Peris-Martínez C. Corneal biomechanical parameters with corneoscleral contact lenses in post-laser in situ keratomileusis eyes. Eye Contact Lens. 2018;44 (Suppl 2):S65-9. doi:10.1097/ICL.0000000000000420

5. Weissman B, Chun MW, Barnhart LA. Corneal abrasion associated with contact lens correction of keratoconus-a retrospective study. Optom Vis Sci. 1994;71(11):677-681. doi:10.1097/00006324-199411000-00001

6. Sengor T, Kurna SA, Aki S, Özkurt Y. High Dk piggyback contact lens system for contact lens-intolerant keratoconus patients. Clin Ophthalmol. 2011;5:331-335. doi:10.2147/OPTH.S16727

7. Walker MK, Schornack MM, Vincent SJ. Anatomical and physiological considerations in scleral lens wear: conjunctiva and sclera. Cont Lens Anterior Eye. 2020;S1367-0484(20)30117-X. doi:10.1016/j. clae.2020.06.005

8. Michaud L, Lipson M, Kramer E, Walker M. The official guide to scleral lens terminology. Cont Lens Anterior Eye. 2019.

9. Koppen C, Kreps EO, Anthonissen L, Van Hoey M, Dhubhghaill SN, Vermeulen L. Scleral lenses reduce the need for corneal transplants in severe keratoconus. Am J Ophthalmol. 2018;185:43-44. doi:10.1016/ j.ajo.2017.10.022

10. Kumar M, Shetty R, Dutta D, Rao HL, Jayadev C, Atchison DA. Effects of a semi-scleral contact lens on refraction and higher order aberrations. Cont Lens Anterior Eye. 2019;42(6):670-674. doi:10.1016/j.clae.2019.06.002

11. Spadea L, Gizzi R, Evangelista Conocchia N, Urbano S. Optical pachymetry-guided custom excimer laser-assisted lamellar keratoplasty for the surgical treatment of keratoconus. $J$ Cataract Refract Surg. 2012;38(9):1559-1567. doi:10.1016/j.jcrs.2012.05.029

12. Krumeich JH, Daniel J, Knulle A. Live-epikeratophakia for keratoconus. $J$ Cataract Refract Surg. 1998;24:456-463. doi:10.1016/S0886-3350(98)80284-8

13. Cacho I, Sanchez-Naves J, Batres L, Pintor J, Carracedo G. Comparison of intraocular pressure before and after laser in situ keratomileusis refractive surgery measured with perkins tonometry, noncontact tonometry, and transpalpebral tonometry. J Ophthalmol. 2015;2015:683895. doi:10.1155/2015/683895

14. Khan JA, LaGreca BA. Tono-pen estimation of intraocular pressure through bandage contact lenses. Am J Ophthalmol. 1989;108 (4):422-425. doi:10.1016/S0002-9394(14)73310-0

15. Sandner D, Bohm A, Kostov S, Pillunat L. Measurement of the intraocular pressure with the "transpalpebral tonometer" TGDc-01 in comparison with applanation tonometry. Graefes Arch Clin Exp Ophthalmol. 2005;243:563-569. doi:10.1007/s00417-004-1037-1

16. Troost A, Yun SH, Specht K, Krummenauer F, Schwenn O. Transpalpebral tonometry: reliability and comparison with Goldmann applanation tonometry and palpation in healthy volunteers. $\mathrm{Br}$ J Ophthalmol. 2005;89:280-283. doi:10.1136/bjo.2004.050211

17. Kim S, Lee JS, Park YK, et al. Fitting miniscleral contact lenses in Korean patients with keratoconus. Clin Exp Optom. 2017;100 (4):375-379. doi:10.1111/cxo.12424

18. Montalt JC, Porcar E, España-Gregori E, Peris-Martínez C. Visual quality with corneoscleral contact lenses for keratoconus management. Cont Lens Anterior Eye. 2018;41(4):351-356. doi:10.1016/j.clae.2018.01.002 
19. Sabesan R, Johns L, Tomashevskaya O, Jacobs DS, Rosenthal P, Yoon G. Wavefront-guided scleral lens prosthetic device for keratoconus. Optom Vis Sci. 2013;90(4):314-323. doi:10.1097/ OPX.0b013e318288d19c

20. Nau CB, Schornack MM, McLaren JW, Sit AJ. Intraocular pressure after 2 hours of small- diameter scleral lens wear. Eye Contact Lens. 2015;42:350-353. doi:10.1097/ICL.0000000000000214

21. Michaud L, Samaha D, Giasson CJ. Intra-ocular pressure variation associated with the wear of scleral lenses of different diameters. Contact Lens Anterior Eye. 2019;42(1):104-110. doi:10.1016/j. clae.2018.07.004

22. McMonnies CW. A hypothesis that scleral contact lenses could elevate intraocular pressure. Clin Experiment Optom. 2016;99 (6):594-596. doi:10.1111/cxo.12368

23. McMonnies CW, Boneham GC. Experimentally-increased intraocular pressure, using digital forces. Eye Cont Lens. 2007;33:124-129. doi:10.1097/01.icl.0000247637.71618.26

24. Shahnazi KC, Isozaki VL, Chiu GB. Effect of scleral lens wear on central corneal thickness and intraocular pressure in patients with ocular surface disease. Eye Contact Lens. 2019. doi:10.1097/ ICL.0000000000000670

25. Kramer EG, Vincent SJ. Intraocular pressure changes in neophyte scleral lens wearers: a prospective study. Cont Lens Anterior Eye. 2020;43:SS1367-9. doi:10.1016/j.clae.2020.05.010

26. Vincent SJ, Alonso-Caneiro D, Collins MJ. Evidence on scleral contact lenses and intraocular pressure. Clin Exp Optom. 2017;100 (1):87-88. doi:10.1111/cxo.12448

27. Aitseabomo AP, Wong-Powell J, Miller W, Farshid A. Effect of scleral lens wear on intraocular pressure. Invest Ophthalmol Vis Sci. 2018;3(1):e1-e9.
28. Turpin S, Antoniuk K, Caroline P, Kojima R, Kinoshita B, Lampa M, Does IOP increase during scleral lens wear? Poster presentation at the 2018 Global Specialty Lens Symposium, Jan 2018, Las Vegas.

29. Cheung SY, Collins MJ, Vincent SJ. The impact of short-term fenestrated scleral lens wear on intraocular pressure. Cont Lens Anterior Eye. 2020;43:SS1367. doi:10.1016/j.clae.2020.02.003

30. McMonnies CW. Re: evidence on scleral contact lenses and intraocular pressure. Clin Exp Optom. 2017;100(2):200. doi:10.1111/ cxo. 12505

31. Toker MI, Vural A, Erdogan H, Topalkara A, Arici MK. Central corneal thickness and Diaton transpalpebral tonometry. Graefes Arch Clin Exp Ophthalmol. 2008;246(6):881-889. doi:10.1007/ s00417-008-0769-8

32. Wisse RPL, Peeters N, Imhof SM, van der Lelij A. Comparison of Diaton transpalpebral tonometer with applanation tonometry in keratoconus. Int J Ophthalmol. 2016;9(3):395-398. doi:10.18240/ ijo.2016.03.12

33. Li Y, Shi J, Duan X, Fan F. Transpalpebral measurement of intraocular pressure using the Diaton tonometer versus standard Goldmann applanation tonometry. Graefes Arch Clin Exp Ophthalmol. 2010;248:1765-1770. doi:10.1007/s00417-009-1243-y

34. Doherty MD, Carrim ZI, O'Neill DP. Diaton tonometry: an assessment of validity and preference against Goldmann tonometry. Clin Exp Ophthalmol. 2012;40(4):e171-e5. doi:10.1111/j.14429071.2011.02636.x

35. Bali SJ, Bhartiya S, Sobti A, Dada T, Panda A. Comparative evaluation of Diaton and Goldmann applanation tonometers. Ophthalmologica. 2012;228(1):42-46. doi:10.1159/000336047
Therapeutics and Clinical Risk Management

\section{Publish your work in this journal}

Therapeutics and Clinical Risk Management is an international, peerreviewed journal of clinical therapeutics and risk management, focusing on concise rapid reporting of clinical studies in all therapeutic areas, outcomes, safety, and programs for the effective, safe, and sustained use of medicines. This journal is indexed on PubMed Central, CAS,

\section{Dovepress}

EMBase, Scopus and the Elsevier Bibliographic databases. The manuscript management system is completely online and includes a very quick and fair peer-review system, which is all easy to use. Visit http://www.dovepress.com/testimonials.php to read real quotes from published authors. 